\title{
Evaluating the Impact of Vaccination on COVID-19 Using Model Checking
}

\author{
Xin Li \\ Shanghai Key Laboratory of Trustworthy Computing \\ East China Normal University, Shanghai, China \\ xli@sei.ecnu.edu.cn
}

\begin{abstract}
Since last year, COVID-19 has become a global issue and brings crisis in nearly every aspect. At the end of last year, COVID-19 vaccines finished developing and began to be large-scale administered. However, the impact of vaccination on COVID-19 is not clear yet. The current vaccine manufacturing capacity is highly insufficient, thus, it is urgent to implement an optimized vaccine prioritization strategy. To shed light on these vaccination issues in our study, we used a fluid model checking method to track the trends of COVID-19 dynamically. First, we proposed a vaccine-related epidemiological model, the SEIHRV model, to investigate the vaccine's impact on the pandemic based on previous studies. Then we embraced a fluid model checking approach to evaluate this SEIHRV model. Compared with the compartmental and agent-based model methods, our approach made a trade-off between speed and accuracy. Lastly, we took several exceptional scenarios into account based on the current COVID-19 situations, including the individual's daily activities, hospital capacity, vaccine prioritization strategy, the variant virus. Our work applied this fluid model checking method to COVID-19 studies, which demonstrates the up-to-date computing method can combine with social concerns and deal with practical problems from an innovative perspective.
\end{abstract}

Index Terms-COVID-19; Vaccine; SEIHRV model; Fluid model checking; Continuous-time Markov-chain

\section{INTRODUCTION}

In the past year of 2020, coronavirus disease 2019 (COVID19) pandemic which is caused by a severe acute respiratory syndrome coronavirus type 2 (SARS-CoV-2) has swept the world, seriously affected and even threatened all aspects of people's lives. As of March 9, 2021, more than 117 million COVID-19 cases have been reported worldwide, of which death cases exceeded 2.6 million (data from Center for Systems Science and Engineering at Johns Hopkins University [1]). In order to reduce the infection rate and mortality rate of COVID-19, governments all around the world have implemented a series of lockdown policies. Researchers in different domains from different countries also actively participate in the COVID-19 studies, especially, in developing the COVID19 vaccine.

Many COVID-19 vaccine projects started since the beginning of 2020, vaccine developed by BioNTech/Pfizer first obtained the emergency use authorization by FDA (U.S. Food and Drug Administration) and European Union on December of 2020. Subsequently,another vaccine developed by Oxford and AstraZeneca [2] was also approved by several European countries. The emergence of vaccines brings the hope of defeating COVID-19 to people. Ideally, if the majority of the population obtain protection from vaccines, COVID-19 reproduction rate would decrease, and it would disappear or be inactivated in the end.

However, there are still some challenges in achieving herd immunity through vaccination. The first challenge is the manufacturing capability. Although vaccine manufacturers have accelerated the large-scale production of vaccines, so far, there are only 300 million doses have been administered worldwide [3]. Due to this situation, countries have to implement various priority strategies to ensure that priority groups (such as the elderly, medical staff, etc.) can be first administered. Nevertheless, these strategies might not be the most efficient one [4]. Second, vaccine efficacy is not completely clear. According to statistics, BioNTech/Pfizer vaccines have an efficacy rate close to 95 percent [5], while Oxford-AstraZeneca vaccines have 82.4 percent for two doses separated by 12 weeks [6]. Third, newly emerged variants SARS-CoV-2 called B.1.1.7 (from the United Kingdom) and B.1.351 (from South Africa) induce increased risk of death and infection, but how they affect vaccine efficacy is still an open question.

Since various factors have brought great uncertainty to the COVID-19 pandemic and the vaccine efficacy, a method that can dynamically monitor the vaccine efficacy is urgently required. Jensen and colleagues used the UPPAAL SMC tool to not only simulate the overall trend of COVID-19 pandemic, but also dynamically track the individual's infection rate and other COVID-19 indicators [7]. Based on their work, we also applied UPPAAL here to check how the Susceptible-ExposedInfected-Hospitalized-Recovered Model (SEIHR model [8]) simulates the COVID-19 pandemic after the large-scale vaccine administration. Here, we considered the latest COVID situations, including the vaccine prioritization strategy [4], the age distribution of COVID cases [9], vaccine efficacy, and vaccine performance against variant viruses. In this way, we can get a comprehensive understanding of how this SEIHR model simulating this pandemic in various situations.

The contributions of this paper are as follows:

- We proposed a vaccine-related epidemiological model to investigate the vaccine's effects on the COVID-19 pandemic.

- We embraced the fluid model checking to analyze the infectious disease model. Compared with the compartmental and the agent-based model method, our approach 
makes a balance between speed and accuracy.

- We demonstrated some complicated situations could be efficiently simulated by our model, such as the individual case situations, hospital capacity, and vaccine prioritization strategy. This approach bridges the gaps between upto-date computing methods and social concerns.

This paper is organized into five sections: Section II introduces the background of epidemiological models and a stochastic model checking method, while Section III describes the widely-used basic reproduction number and our SEIHRV model. Section IV emphasizes our experiment and discussion. Section V draws the conclusion.

\section{BACKGROUND}

\section{A. The Compartmental Model in Epidemiology}

In epidemiology, Kermack and McKendrick proposed a dynamic compartmental SIR model to simulate the influences of an infectious disease [8]. The original SIR model divided individuals into three groups depending on their physical states (compartments): susceptible (S), infectious(I), and recovered (R) group. It assumes that all individuals are initially susceptible, while all recovered individuals will have antibodies and will not be re-infected again. The dynamical flow of this SIR model can be represented as follows:

$$
\mathcal{S} \rightarrow \mathcal{I} \rightarrow \mathcal{R}
$$

The SIR model updates the variables for every time point. $N$ denotes total number of the population, $\beta$ is the fraction of susceptible persons get infected, and $1 / \gamma$ implies the infectious period. The model with transmission is:

$$
\frac{d S}{d t}=-\frac{\beta S I}{N} \quad \frac{d I}{d t}=\frac{\beta S I}{N}-\gamma I \quad \frac{d R}{d t}=\gamma I
$$

The establishment of this dynamical model sheds light on theoretical and quantitative analysis of infectious diseases [10]. It is based on the disease's occurrence, the spread, the transmission rules, and related social factors. Through qualitative, quantitative analysis, and numerical simulation, it is possible to track where the disease comes from, monitor some key elements, and even predict the disease trends.

Using the continuous-time ordinary differential equation (ODE) method, this traditional compartmental model can easily simulate the general population's epidemic development. Nevertheless, it does not allow the prediction for individuals, how compartment changed with individuals' activities. Moreover, it is impossible to describe some exceptional cases, like the variant COVID-19 virus and the super spreaders. To simulate these complicated cases, a fluid model checking is necessary.

\section{B. Stochastic Model Checking}

Stochastic model checking is an extension of classical model checking theory, serves as an automatic model-based formal verification of stochastic systems [11]. In recent years, stochastic model testing has attracted a lot of attention in the formal verification domain and has made significant progress. It has been applied to the verification of probabilistic programs, system performance analyses, communication protocol reliability analyses, service quality optimization of service processes, and even computational biology [12]. Typically, the continuous-time Markov chain (CTMC) [13] is used to represent models in stochastic model checking, CTMC is a tuple $\left(S, R, \pi_{0}\right)$ where:

- $S$ is the set of states,

- $\mathrm{R}: S \times S \rightarrow \mathbb{R} \geq 0$ is the transition rate matrix, and

- $\pi_{0}$ is the initial distribution.

We defined the exit rate of s state $s \in S$ as

$$
E(s)=\sum_{s^{\prime} \in S} \mathrm{R}\left(s, s^{\prime}\right)
$$

The embedded DTMC coincides on $S$ and $\pi_{0}$ but has the transition probability matrix $E$. It can be defined as:

$$
E\left(s, s^{\prime}\right)=\left\{\begin{array}{lr}
\frac{\mathrm{R}\left(s, s^{\prime}\right)}{E(s)} & \text { if } E(s)>0 \\
0 & \text { if } E(s)=0 \wedge s \neq s^{\prime}, \text { and } \\
1 & \text { if } E(s)=0 \wedge s=s^{\prime} .
\end{array}\right.
$$

The probability measure for a CTMC $\left(S, R, \pi_{0}\right)$ is induced by the measure for cylinder sets $P\left(C\left(s_{0} / 0 \ldots s_{n}\right)\right)$ defined as:

$$
\pi_{0}(s) \prod_{0 \leq i<n} \mathrm{E}\left(s_{i}, s_{i+1}\right)\left(e^{-E\left(s_{i}\right) \inf l_{i}}-e^{-E\left(s_{i}\right) \sup l_{i}}\right) .
$$

The stochastic model checking algorithm combines the classical model checking algorithm and linear equation system solution or linear programming algorithm. Its operation deals with the probability vector about the state instead of the bit vector in the classical model checking. However, the stochastic model checking is faced with a more challenging problem,the state-space explosion problem. It is still not clear how to mitigate the state explosion problem for large-scale stochastic systems and apply stochastic model testing to the quantitative verification and analysis of such systems, even Turing Award winner Clarke listed this as a crucial direction for future research in model testing in his Turing Lecture [14]. Researchers recently found that fluid model checking, a combination of ODE and CTMC, can efficiently mitigate the state-space explosion [15].

\section{BASIC REPRODUCTION NUMBER AND SEIHRV MODEL}

MacDonald and colleagues established a mathematical model on the spread of an epidemic based on malaria studies [16]. There is a crucial indicator in their model, the basic reproduction number $R_{0} . R_{0}$ that greater than 0 indicates this epidemic will continue to spread; otherwise, this epidemic will eventually be eliminated. The reproduction number has been widely used in the epidemiological domain, and now it becomes one of the most valuable indicator in COVID-19 studies [17]. 
In our paper, a variant of the SIR model: the SEIHRV model was defined. We also assumed all individuals are initially susceptible (S), they become exposed (E) once they contact with an infectious people, the transition rate can be denoted as $\lambda$. After a latency period ( $d_{E}$ days), the exposed individual will transition to an infectious individual (I). Those infectious people with severe symptoms for few days $\left(d_{I H}\right)$ are accepted to the hospital $(\mathrm{H})$ (HR: hospitalization rate), the majority of them will recover $(\mathrm{R})$ after several days $\left(d_{H}\right)$ treatment, a small proportion (IFR: infection fatality rate) would die even been treated. Meanwhile, most infectious people will spend some days $\left(d_{I R}\right)$ to eventually recover $(\mathrm{R})$ by relying on their self-immunity. In this model, $\lambda$ is obtained by:

$$
\lambda=u \sum_{j} c_{j} \frac{I}{N-\Omega}
$$

where $u$ is the probability of a successful transmission from an infectious contact and the $c_{j}$ is the number of individuals that a susceptible individual contacts per day. $I /(N-\Omega)$ refers to the probability that a random individual is infectious, where $N$ is the total population number, $I, \Omega$ is the infectious number and death number, respectively. $\lambda$ can also predict basic reproduction number $R_{0}$.

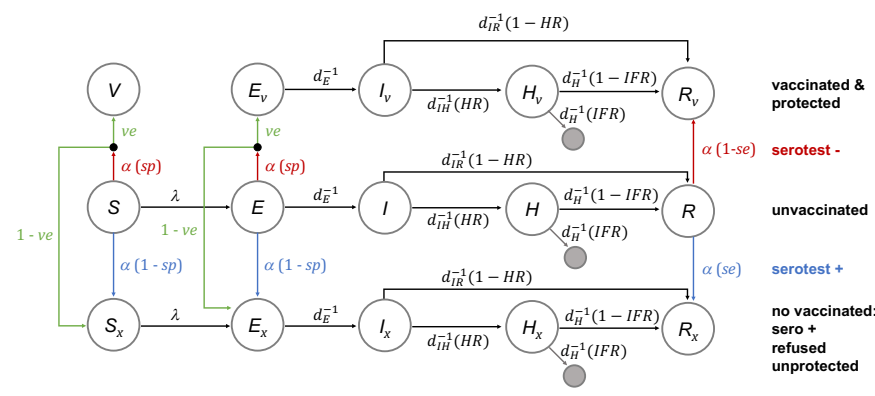

Fig. 1. Schematic diagram of a dynamic SEIHRV model with vaccination.

To evaluate the development of the COVID-19 pandemic after large-scale vaccine administration, we added vaccination effect in the original SEIHR model and named the new model as SEIHRV model. We divided the whole population into three groups as previous work did [4]: people were vaccinated and effectively protected by the vaccine, unvaccinated people (can be vaccinated), and people ineligible for vaccination, which includes vaccine-hesitant people, people with positive serotest result or vaccinated people without protection. The schematic diagram is shown in Fig. 1. Whether an individual can be administrated or whether a vaccinated person is perfectly protected largely depend on the serological test results. However, the serological test is not entirely accurate. To illustrate this constraint, we also took the sensitivity $(s e)$ and the specificity $(s p)$ of the serological test into consideration. $\alpha$ in this model represents the vaccine rollout rate in susceptible and exposed populations, and $\alpha=n_{\mathrm{vax}} /[(S+E) s p+R(1-s e)]$, where $n_{\mathrm{vax}}$ indicates the amount of vaccines to be rolled out in a single day. $v_{e}$ denotes the vaccine efficacy. A small proportion of the susceptible people and the exposed population with negative serotest results can be vaccinated $(\alpha(s p))$ per day. Some of them are well protected by the vaccine and will be members of $V$ or $E_{v}$ group, the rest of the people who are ineligible for vaccination or without vaccine protection will be grouped into $S_{x}$ and $E_{x}$. The following equations describe the compartment-to-compartment relationships:

$$
\begin{aligned}
& S \rightarrow S_{v}=\frac{n_{\mathrm{vax}}}{(S+E) s p+R(1-s e)} S(s p) \\
& E \rightarrow E_{v}=\frac{n_{\mathrm{vax}}}{(S+E) s p+R(1-s e)} E(s p) \\
& R \rightarrow R_{v}=\frac{n_{\mathrm{vax}}}{(S+E) s p+R(1-s e)} R(1-s e) \\
& S \rightarrow S_{x}=\frac{n_{\mathrm{vax}}}{(S+E) s p+R(1-s e)} S(1-s p) \\
& E \rightarrow E_{x}=\frac{n_{\mathrm{vax}}}{(S+E) s p+R(1-s e)} E(1-s p) \\
& R \rightarrow R_{x}=\frac{n_{\mathrm{vax}}}{(S+E) s p+R(1-s e)} R(\mathrm{se})
\end{aligned}
$$

\section{EXPERIMENT AND DISCUSSION}

In this section, we first built a continuous-time ordinary differential equation model of SEIHRV, and simulated the 365 day dynamic model corresponding to the first-year vaccination phase. Then we used the continuous-time Markov chain models since they have better performance than ODE if we take individuals' behaviors and decisions into consideration, such as staying at home or wearing a mask. Usually, such models require a large volume of individual activity data and a considerable amount of computational resources.

To improve these, we got the inspirations from the fluid model checking method, combined the ODE and CTMC, to simulate the COVID-19 epidemic. By doing this, the system required less time in performing verification significantly. Thus, we can not only use ODE to describe the population epidemic development, but also use CTMC to describe the individuals' status. Our simulations indicate lockdown and conditional lockdown are the best options for reducing infection fatality rate and virus transmission rate.

Additionally, we simulated the emerging cases caused by the variant and emphasized how the variant affects hospital capacity. Lastly, we focused on the current vaccine prioritization strategy and predicted the trends of COVID-19 after vaccination. These simulations and models can provide beneficial assistance to COVID-19 researches and policy formulation.

\section{A. Ordinary differential equation models}

In the first part, we established the ODE model of SEIHRV in UPPAAL SMC [18]. All individuals in this model are initially susceptible unless they have been effectively vaccinated or have already got natural immunity. The recovered individuals are no longer infectious in the simulation period (up to 365 days) and cannot be re-infected. Details of SEIHRV model can be found in Section III.

As shown in Fig. 2, only one state was required for one individual to build the above ODE model system in UPPAAL SMC, and all of the populations were represented by using the variable CLOCK in UPPAAL SMC. 


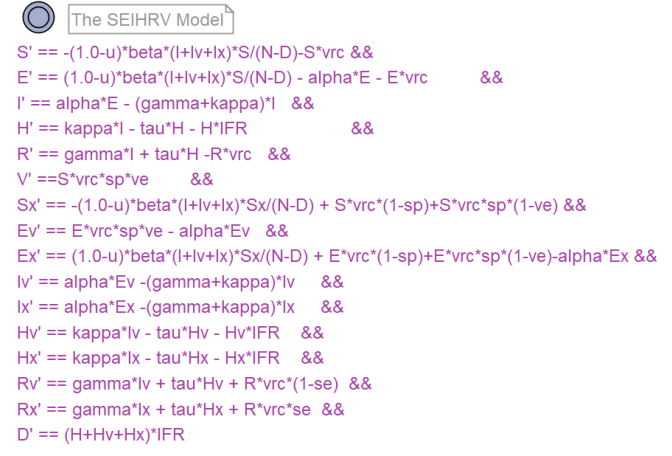

Fig. 2. ODE model of SEIHRV

We hypothesized a town with a 10,000 population in our simulation, the basic reproduction number of this town is 1.3 , and $1 \%$ people have been infected. Other demographic data, such as infection and fatality rate, were obtained from CDC [1]. Meanwhile, we assumed the vaccine rollout rate is $0.2 \%$ per day, and the vaccine efficacy is $95 \%$, which means vaccines are not one hundred percent efficient. The specificity and sensitivity value of a serological test is 0.97 and 0.99 .

TABLE I

QUERY OF EPIDEMIC TREND

\begin{tabular}{ll}
\hline Purpose & Query \\
\hline Epidemic trend & simulate $[<=365]\{S, E, I, H, R, V, D\}$ \\
\hline
\end{tabular}

Next, a query was described in UPPAAL SMC. As shown in TABLE I, we counted the susceptible, exposed, infectious, hospitalized, recovered, and dead people within 365 days and analyzed COVID-19 trends.

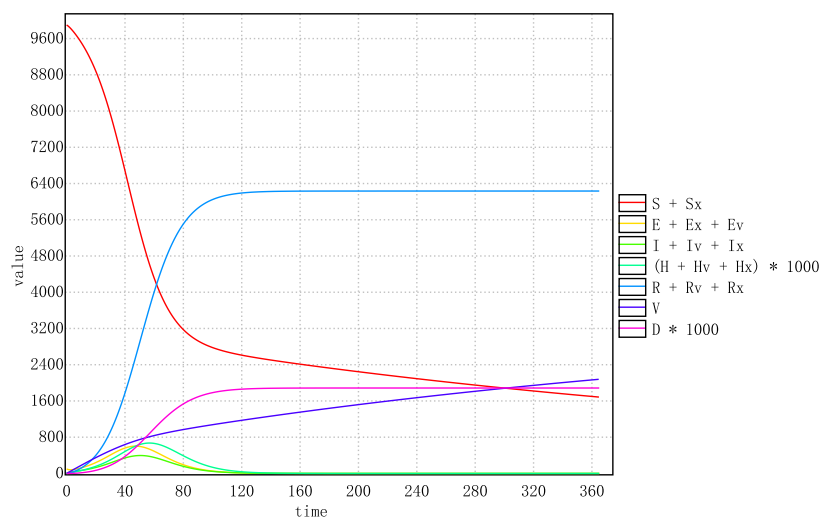

Fig. 3. Evolution of the epidemic with vaccine

Simulation results indicate this epidemic is hard to control at the very beginning of the vaccination. However, the situation will become better once the number of vaccinated population increases, the transmission rate will simultaneously decrease (Fig. 3). Around 60 days after vaccination, the susceptible line and the recovered line will intersect, the inflection point of the epidemic appear, which means the epidemic is gradually under-controlled.

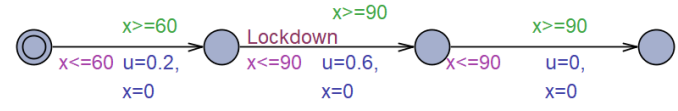

(a) Lockdown mechanism

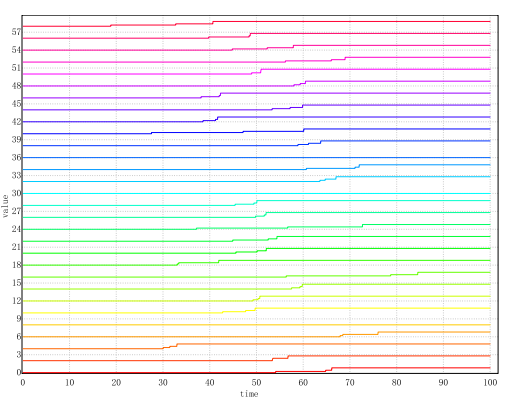

(b) Health conditions of 30 persons

Fig. 4. Model and Result

B. Continuous-time Markov chain models and Fluid model checking

Although the ODE model in the previous section can describe the epidemic trends at the population level, it cannot describe individuals' behavior and incorporate some other details, like the age-dependent infection rate and fatality rate, the lockdown, etc. Therefore, we built the CTMC model of SEIHRV here to involve detailed information (Fig. 5):

In this model, each location has one corresponding exponential distribution function, and different weights were given to different edges. For example, in Fig. 5, location $I$ has different probabilities to location $H$ or $L$. Initially, we assumed a CTMC could describe an individual's status, and used the 10,000 population case to test it. However, we found this operation occupied massive computing resources, since 10,000 CTMC models are required to generate based on the template and simultaneously run. Moreover, the state-space of the system increased exponentially as the number of people increased, and the state-space explosion problem appeared.

Therefore, in order to efficiently model these data with individual information, we adopted the fluid model checking method in our experiment, abstracted the population epidemic situation, and only focused on the target groups. Combination of the ODE model and the CTMC model in UPPAAL SMC can significantly reduce the memory and time required for simulation and verification.

We simulated a lockdown scenario here and found transmission significantly decreased in the lockdown period (Fig. 4(a)). Furthermore, we selected 30 specific individuals in this model and analyzed their health conditions (Fig. 4(b)). If their health conditions varied largely, their personal curves would also be fluctuating. The above simulation took a long time if we only used the CTMC model. However, the simulation time shortened to one-tenth if the CTMC model was used to represent 30 target individuals and others are modeled by ODE. Thus, the fluid model checking significantly reduced the memory and time required for simulation and verification. 


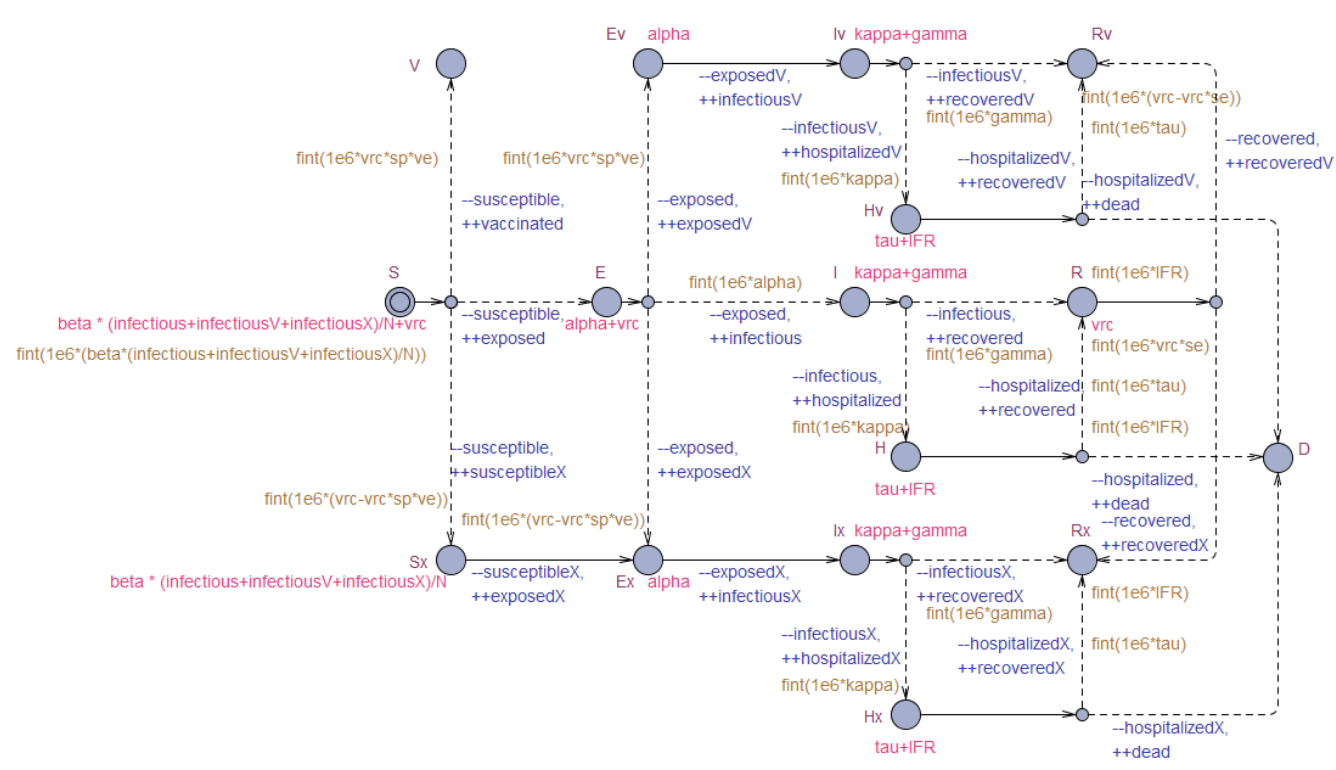

Fig. 5. CTMC model of SEIHRV

\section{Hospital capacity and Variant of SARS-CoV-2}

A variant of the SARS-CoV-2 was first identified in UK in September 2020, and then it spreads around the world in the next few months. Studies show this variant has a 40-70\% higher transmission rate than the original virus [19]. By far, it has been found in more than 100 countries and takes Germany as an example, the variant infected nearly $55 \%$ of the cases. Studies demonstrated this variant could also induce a 30-100\% higher infection fatality rate than previous viruses.

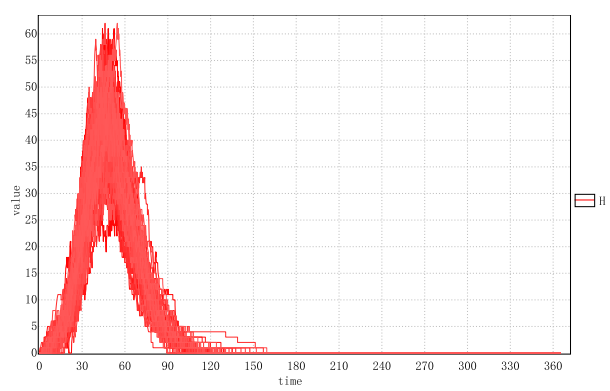

Fig. 6. Evolution of hospitalized

To include this variant in our model, we accordingly modified our parameters. In the updated model, $55 \%$ of patients were infected by the new variant. Furthermore, due to the high infection rate and fatality rate caused by this variant, we addressed hospital capacity here. Once the number of patients with severe symptoms exceeds hospitals' capacity, the fatality rate would rise rapidly. So we had the following query in TABLE II, the query "Hospitalized trend" can be explained as to how the number of hospitalized population changes.

One hundred hospitalized cases were simulated here, data shows hospitalization peak occurs at around 40 days after vaccination, and the number of hospitalized patients is about 60 (Fig. 6). Those simulation data can help medical institu-
TABLE II

QUERY OF HOSPITALIZED

\begin{tabular}{ll}
\hline Purpose & Query \\
\hline Hospitalized trend & simulate $[<=365 ; 100]\{\mathrm{H}\}$ \\
Hospital capacity & $\operatorname{Pr}[<=365](<>\mathrm{H}>=60)$ \\
\hline
\end{tabular}

tions monitor the hospitalization load dynamically and make complete preparations in advance.

To get more details, we used the query "Hospital capacity" to calculate the probability of hospitalized cases exceed 60 . As shown in Fig. 7, the range is [0.0125218,0.112309] with confidence 0.95 . Thus, we suggest medical institutions to pay sufficient attention to this variant, it could bring vast pressure on the medical system.

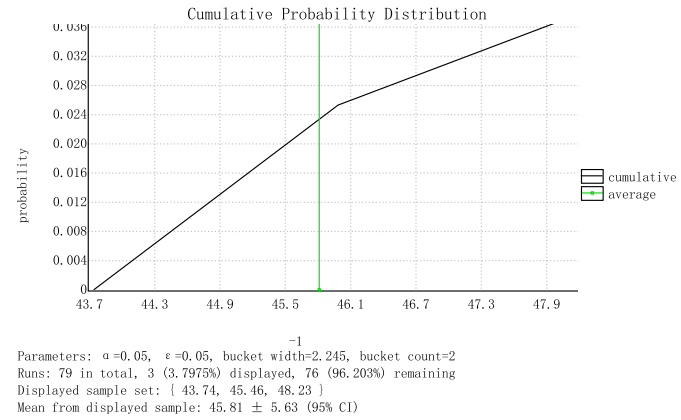

Fig. 7. Probability Distribution

\section{Vaccine prioritization strategy}

At the beginning of large-scale vaccination, vaccines' manufacturing capability is limited and it is still hard to achieve herd immunity, optimizing the vaccine prioritization strategy has become a crucial issue. Most prioritization strategy is agedependent since infection rate and infection fatality rate are closely associated with ages. In this section, we applied the 
fluid checking model to analyze various vaccine prioritization strategies.

Here, three different vaccines were used: Pfizer-BioNTech, Moderna, and Oxford/AstraZeneca. Their efficacies are 95\% and $94.1 \%$ and $82.4 \%$, respectively. We used the age demographic data from UN [20] to estimate the age distribution, and grouped individuals by their ages with 10-year increment steps. Some other age-stratified values from previous literature [9] served as model parameters here, which include infection rate, fatality rate, hospitalization rate, and recovery period.

Four age-dependent vaccine prioritization strategies: young people under 20 years old, middle-aged and young adults between 20 and 49 years old, old people over 60 years old, and all people over 20 years old, were simulated by using our previous approach.

We assumed all remaining individuals have equal priority once the vaccination for the priority group has been completed. Here we present simulations of 20-49 (Fig. 8(b)) and 60+ (Fig. 8 (a)) prioritization strategy (Pfizer-BioNTech).

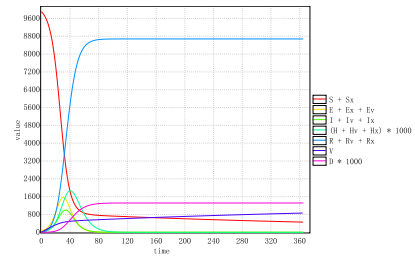

(a) Adults $60+$ first

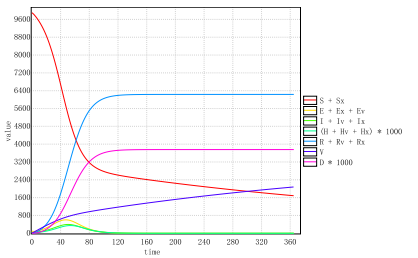

(b) Adults 20-49 first
Fig. 8. Simulation results

20-49 vaccine prioritization strategy effectively lowers down the infection rate and transmission rate (Fig. 8(b)). Those results are reasonable because individuals at that age tend to have more social activities, most of them work everyday and are more likely to contact infectious individuals. Meanwhile, $60+$ strategy can effective reduce the fatality rate (Fig. 8 (a)) mainly because old people usually have higher hospitalization rates and fatality rates once they are infected. Since every vaccine prioritization strategy has its own benefits, it is crucial to make a trade-off between different COVID-19 vaccine prioritization strategies.

\section{CONCLUSION}

In this paper, we used a fluid model checking method to conduct a series of studies on the current COVID-19 pandemic. First, we proposed a vaccine-related epidemiological model: the SEIHRV model. With this model, we are able to investigate the vaccination impact on the current pandemic more rapidly, efficiently, and flexibly. Second, the fluid model checking method was embraced to model, simulate, verify, and analyze this SEIHRV model. We elaborated this method in detail and compared it with the traditional compartmental model method. Results show it performed better in complicated scenario simulations. Moreover, this method is adjustable on different individual's specific conditions and can provide more detailed information. In comparison with the agent-based method, fluid model checking can effectively reduce computing resources' requirements and lessen the state space explosion. Finally, based on the true-to-life situations, we simulated the cases individually, also how the hospital capacity, variant virus, and vaccine prioritization strategy affect the COVID-19 trends. In conclusion, this approach provide a more practical and efficient way to simulate COVID-19 development in complicated scenarios, especially after the large-scale vaccine administration. Correspondingly, its simulation results can provide more comprehensive and detailed information on COVID-19.

\section{REFERENCES}

[1] S. Science and E. at Johns Hopkins University, "Covid-19 dashboard," gisanddata.maps.arcgis.com/apps/opsdashboard.

[2] M. D. Knoll and C. Wonodi, "Oxford-astrazeneca covid-19 vaccine efficacy," The Lancet, vol. 397, no. 10269, pp. 72-74, 2021.

[3] "Data on covid-19 (coronavirus) vaccinations by our world in data," github.com/owid/covid-19-data/tree/master/public/data/vaccinations.

[4] K. M. Bubar, K. Reinholt, S. M. Kissler, M. Lipsitch, S. Cobey, Y. H. Grad, and D. B. Larremore, "Model-informed covid-19 vaccine prioritization strategies by age and serostatus," Science, vol. 371, no. 6532, pp. 916-921, 2021.

[5] E. Mahase, "Covid-19: Pfizer vaccine efficacy was 52\% after first dose and $95 \%$ after second dose, paper shows," 2020.

[6] M. Voysey, S. A. C. Clemens, S. A. Madhi, L. Y. Weckx, P. M. Folegatti, P. K. Aley, B. Angus, V. L. Baillie, S. L. Barnabas, Q. E. Bhorat et al., "Single-dose administration and the influence of the timing of the booster dose on immunogenicity and efficacy of chadox 1 ncov-19 (azd1222) vaccine: a pooled analysis of four randomised trials," The Lancet, 2021.

[7] P. G. Jensen, K. Y. Jørgensen, K. G. Larsen, M. Mikučionis, M. Muñiz, and D. B. Poulsen, "Fluid model-checking in uppaal for covid-19," in International Symposium on Leveraging Applications of Formal Methods. Springer, 2020, pp. 385-403.

[8] W. O. Kermack and A. G. McKendrick, "Contributions to the mathematical theory of epidemics. ii.-the problem of endemicity," Proceedings of the Royal Society of London. Series A, containing papers of a mathematical and physical character, vol. 138, no. 834, pp. 55-83, 1932.

[9] N. G. Davies, P. Klepac, Y. Liu, K. Prem, M. Jit, and R. M. Eggo, "Agedependent effects in the transmission and control of covid-19 epidemics," Nature medicine, vol. 26, no. 8, pp. 1205-1211, 2020.

[10] G. Giordano, F. Blanchini, R. Bruno, P. Colaneri, A. Di Filippo, A. Di Matteo, and M. Colaneri, "Modelling the covid-19 epidemic and implementation of population-wide interventions in italy," Nature medicine, vol. 26, no. 6, pp. 855-860, 2020.

[11] M. Kwiatkowska, G. Norman, and D. Parker, "Stochastic model checking," in International School on Formal Methods for the Design of Computer, Communication and Software Systems. Springer, 2007, pp. 220-270.

[12] M. Kwiatkowska, G. Norman, and D. Parker, "Using probabilistic model checking in systems biology," ACM SIGMETRICS Performance Evaluation Review, vol. 35, no. 4, pp. 14-21, 2008.

[13] A. Aziz, K. Sanwal, V. Singhal, and R. Brayton, "Model-checking continuous-time markov chains," ACM Transactions on Computational Logic (TOCL), vol. 1, no. 1, pp. 162-170, 2000.

[14] E. M. Clarke Jr, O. Grumberg, D. Kroening, D. Peled, and H. Veith, Model checking. MIT press, 2018.

[15] L. Bortolussi and J. Hillston, "Fluid model checking," in International Conference on Concurrency Theory. Springer, 2012, pp. 333-347.

[16] G. Macdonald, "The measurement of malaria transmission," 1955.

[17] R. Subramanian, Q. He, and M. Pascual, "Quantifying asymptomatic infection and transmission of covid-19 in new york city using observed cases, serology, and testing capacity," Proceedings of the National Academy of Sciences, vol. 118, no. 9, 2021.

[18] G. Behrmann, A. David, K. G. Larsen, J. Håkansson, P. Pettersson, W. Yi, and M. Hendriks, "Uppaal 4.0," 2006.

[19] R. Challen, E. Brooks-Pollock, J. M. Read, L. Dyson, K. TsanevaAtanasova, and L. Danon, "Risk of mortality in patients infected with sars-cov-2 variant of concern 202012/1: matched cohort study," BMJ, vol. $372,2021$.

[20] U. Nations, "Age demographic data," population.un.org/wpp/Download/ Standard/Population/. 